\title{
FERMIONS ON HALF-QUANTUM VORTEX
}

\author{
G.E. Volovik \\ Low Temperature Laboratory \\ Helsinki University of Technology \\ Otakaari 3A, 02150 Espoo, Finland \\ and \\ L.D. Landau Institute for Theoretical Physics, \\ Kosygin Str. 2, 117940 Moscow, Russia
}

August 18, 2021

\begin{abstract}
The spectrum of the fermion zero modes in the vicinity of the vortex with fractional winding number is discussed. This is inspired by the observation of the $1 / 2$ vortex in high-temperature superconductors [1]. The fractional value of the winding number leads to the fractional value of the invariant, which describes the topology of the energy spectrum of fermions. This results in the phenomenon of the "halfcrossing": the spectrum approaches zero but does not cross it, being captured at the zero energy level. The similarity with the phenomenon of the fermion condensation is discussed.
\end{abstract}




\section{Introduction}

Recently a new topological object in high-temperature superconductor - the vortex with the fractional winding number $N=1 / 2$ - has been observed [四]. This 1/2-vortex was predicted in Ref.[2].

As a rule, the fractional vortices are coupled to the topological or nontopological surfaces: In ${ }^{3} \mathrm{He}-\mathrm{A}$ where such vortices should exist [3] but still have not been identified, they represent the termination lines of the topological $Z_{2}$-solitons. The same occurs for the $1 / 2$ vortices discussed in heavy fermionic systems [4]. In high-temperature superconductors the observed $1 / 2$ vortex is attached to the tricrystal line, which is the junction of three grain boundaries [2, 1]. In ${ }^{3} \mathrm{He}-\mathrm{B}$ a pair of $1 / 2$ vortices, connected by the nontopological wall, forms a nonaxisymmetric $N=1$ vortex [5, 6, 7], the latter vortex has been experimentally identified in Ref. [8]. In Ref. [9] even the 1/4 quantum vortices were predicted to exist in ${ }^{3} \mathrm{He}-\mathrm{A}_{2}$, they are connected by the nontopological walls discussed in $[10$.

The $N=1 / 2$ vortex observed in high-temperature superconductor carries $1 / 2$ of the magnetic flux quantum $\Phi_{0}=h / 2 e$ [1]. In principle the flux number does not necessarily coincide with the winding number $N$, thus the objects with the fractional flux below $\Phi_{0} / 2$ are also possible [11]. They can arise if the time inversion symmetry is broken 12, 13.

One may expect different interesting phenomena related to the behavior of the quasiparticles (fermions and bosons) in the presence of fractional vortices. These vortices are the counterpart of Alice strings, which appear in some models of particle physics: the particle travelling around some type of the Alice string changes its electric charge to the opposite [14. In ${ }^{3} \mathrm{He}-\mathrm{A}$, the analogous effect is the reversal of the spin of the quasiparticle upon circling the $1 / 2$ vortex. This behavior results also in the peculiar Aharonov-Bohm effect, which has been discussed for $1 / 2$ vortices in ${ }^{3} \mathrm{He}-\mathrm{A}$ [15, 6] and has been modified for the cosmic Alice strings in [16, 17.

Here we discuss the behavior of the fermion zero modes - Caroli-de Gennes-Matricon (CGM) bound states [18] in the core of vortices. As was found in Ref. 19] there is some kind of the index theorem for the CGM levels. It states that the number of branches of the quasiparticle spectrum, which as function of the angular momentum $Q$ cross the zero energy, equals the winding number $N$ per each of two spin projections. For the conventional $N=1$ vortex in $s$-wave superconductors this results in two branches of fermion zero 
modes corresponding to two spin projections. The problem is what happens if the winding number per spin is fractional. This is the relevant case for the $N=1 / 2$ vortices in heavy fermionic and high-T superconductors and for the $N=1 / 4$ vortices in ${ }^{3} \mathrm{He}-\mathrm{A}_{2}$. (This is not related to the $1 / 2$ vortex in

${ }^{3} \mathrm{He}-\mathrm{A}$, because ${ }^{3} \mathrm{He}-\mathrm{A}$ can be effectively represented as an equal mixture of spin-up and spin-down components, and the $1 / 2$ vortex corresponds to the $N=1$ vortex in one component with $N=0$ for another component. Thus the winding number per spin appears to be integer for each component.)

Here we shall use the model of $1 / 2$ vortex discussed in 4 . The result is schematically presented in Fig.1. While for the $N=1$ vortex the spectrum $E(Q)$ crosses zero (Fig.1a), for the $N=1 / 2$ vortex the spectrum instead of crossing asymptotically approaches zero (Fig.1b). The Fig.1c shows the spectrum for the case when two $1 / 2$ vortices together form the $N=1$ vortex.

\section{2 "Half-crossing".}

Let us consider the unconventional superconductivity of the $E_{1 g}$ representation, which corresponds to the $d$-wave pairing. The symmetry class which gives rise to the $1 / 2$ vortex is characterized by the following gap function:

$$
\Delta(\mathbf{k}, \mathbf{r})=\Delta_{0}(\mathbf{r}) e^{i \Phi(\mathbf{r})} \hat{k}_{z} \hat{\mathbf{k}} \cdot \hat{\mathbf{d}}(\mathbf{r})
$$

where $\hat{\mathbf{k}}$ is the unit vector along the linear momentum $\mathbf{k}$ of the quasiparticle; the unit real vector $\hat{\mathbf{d}}$ of the spontaneous anisotropy, the condensate phase $\Phi$ and the gap amplitude $\Delta_{0}$ are coordinated dependent in the presence of the $1 / 2$ vortex. We assume the simplest form for the $1 / 2$ vortex with

$$
\Delta_{0}(\mathbf{r})=\Delta_{0}(r), \quad \Phi(\mathbf{r})=\frac{\phi}{2} \quad, \quad \hat{\mathbf{d}}(\mathbf{r})=\hat{x} \cos \frac{\phi}{2}+\hat{y} \sin \frac{\phi}{2}
$$

where $z, r, \phi$ are the cylindrical coordinates. The changes of the sign of the gap function due to the $\pi$ winding of the phase $\Phi=\phi / 2$ is compensated by the change of sign of the $\hat{\mathbf{d}}$ field.

The fermionic spectrum is obtained from the Bogoliubov Hamiltonian, which for the given spin projection is $2 \times 2$ matrix

$$
\mathcal{H}=\hat{\tau}_{3} \varepsilon(\mathbf{p})+\hat{\tau}_{1} \operatorname{Re} \Delta(\mathbf{p}, \mathbf{r})-\hat{\tau}_{2} \operatorname{Im} \Delta(\mathbf{p}, \mathbf{r})
$$


where $\hat{\tau}_{a}$ are the Pauli matrices, $\mathbf{p}=-i \vec{\nabla}, \varepsilon(\mathbf{p})=\left(p^{2}-p_{F}^{2}\right) / 2 m$.

In quasiclassical approximation $\left(p_{F} \xi \gg 1\right.$ where $\xi$ is the core size) the relevant variables are the transverse momentum $\mathbf{q}$ of the incident quasipartice with $q^{2}=p_{F}^{2}-p_{z}^{2}$ and its impact parameter $b$. The angular momentum of the quasipartice is $Q=q b$. For the Ansatz (2) there is an important difference between positive and negative values of the impact parameter $b$, see Fig.2. For one sign of $b$ the quasiparticle trajectory always crosses the surface at which the classical energy takes zero value: $E(\mathbf{k}, \mathbf{r})=\sqrt{\varepsilon^{2}(\mathbf{k})+|\Delta(\mathbf{k}, \mathbf{r})|^{2}}=$ 0 , while for the opposite sign of $b$ the energy is always nonzero. This leads to the asymmetry of the fermionic spectrum in Fig.1b.

Let us consider the simplest example of $\mathbf{q}=q \hat{y}$. In this case the surface of the zero value of the gap in Eq.(1) is the half-plane $y=z=0, x>0$ (see Fig.2). The Hamiltonian is

$$
\mathcal{H}=-i \hat{\tau}_{3} \frac{q}{m} \nabla_{y}+\frac{q k_{z}}{2 p_{F}^{2}} \Delta_{0}(r)\left[\hat{\tau}_{1} \sin \phi-\hat{\tau}_{2}(1-\cos \phi)\right] .
$$

If $b>0$ and large enough, then in the vicinity of the cross point of the trajectory with the zero-energy surface one has $\phi \approx y / b \ll 1$ and the third term can be considered as small perturbation. The first two terms have the normalizable zero-energy solution, which for $b \gg \xi$ has the form

$$
\Psi^{(0)}(y) \propto\left[1-\hat{\tau}_{2} \operatorname{sign}\left(k_{z} b\right)\right] e^{-\frac{m\left|k_{z}\right| \Delta_{0}(\infty)}{4 b p_{F}^{2}} y^{2}} .
$$

The third term averaged over this wave function gives the following energy spectrum:

$$
E(q, b) \sim \frac{q}{m b}, \quad b>0 .
$$

Since the effective $q$ is on order of $p_{F}$, the spectrum in terms of the angular momentum $Q=q b$ has the following form

$$
E_{\mathbf{q}=q \hat{y}}(Q) \sim \frac{E_{F}}{Q}, b>0, b \gg \xi,
$$

where $E_{F}=p_{F}^{2} / 2 m$ is the Fermi-energy. At large $b$ this spectrum approaches the zero energy level: these asymptotic midgap states far from the halfquantum vortex reflect the property of the fermionic bound states in the $\pi$-soliton or in the Josephson junction with the phase difference $\pi$, which emanates from the $1 / 2$ vortex. 
If $b<0$ the trajectory does not cross the zero value of the classical energy and the behavior of the spectrum follows that for the conventional vortex. This can be seen on the model example of the large core size compared with $\xi$. In this case the Hamiltonian in Eq.(4) can be expanded near the point $r=|b|, \phi=0$ :

$$
\mathcal{H}=-i \hat{\tau}_{3} \frac{q}{m} \nabla_{y}+\frac{q k_{z}}{2 p_{F}^{2}} \Delta_{0}(b)\left(\hat{\tau}_{1} \frac{y}{|b|}-2 \hat{\tau}_{2}\right) .
$$

This gives the energy spectrum

$$
E(q, b)=\frac{q\left|k_{z}\right|}{p_{F}^{2}} \Delta_{0}(b), \quad b<0,
$$

and for small $b$, when $\Delta_{0}(b)=|b| \Delta^{\prime}$ the ordinary linear dependence on the angular momentum is obtained

$$
E_{\mathbf{q}=q \hat{y}}(Q) \sim-Q \frac{\Delta_{0}^{2}}{E_{F}}, \quad b<0, \quad|b|<\xi .
$$

Together with the Eq.(7) this gives the spectrum in Fig.1b. For the opposite direction of the transverse momentum $\mathbf{q}=-q \hat{y}$ the spectrum is inverted, see the right part of Fig.1c. Thus when two $N=1 / 2$ vortices form the $N=1$ vortex the topology of the spectrum in Fig.1a is restored: spectra in Fig.1a and Fig.1c have the same topology, which corresponds to one branch crossing zero. If one considers the other orientations of $\mathbf{q}$ one obtains either the spectrum in Fig.1b or the inverted one. Thus for each $\mathbf{q}$ the energy spectrum $E_{\mathbf{q}}(Q)$ in terms of the angular momentum $Q$ "half-crosses" zero.

\section{Relation to the index theorem.}

The "half-crossing" is also supported by the index theorem and corresponds to the fractional value of the topological invariant introduced in [19]. The number of the anomalous branches of the spectrum $E_{\mathbf{q}}(Q)$, which at given $\mathbf{q}$ cross zero as a fuction of $Q$, coincides with the number of the point zeroes of the Bogoliubov Hamiltonian in its classical limit. For $\mathbf{q}=q \hat{y}$ the classical expression follows from Eq.(4)

$$
\mathcal{H}_{\text {class }}\left(p_{y}, y, x\right)=\hat{\tau}_{3} \frac{q}{m} p_{y}+\frac{q k_{z}}{2 p_{F}^{2}} \Delta_{0}(r)\left[\hat{\tau}_{1} \sin \phi-\hat{\tau}_{2}(1-\cos \phi)\right]
$$


which can be written in terms of the unit vector $\hat{m}(\vec{s})$ in the 3 -d space of parameters $\vec{s}=\left(p_{y}, x, y\right)$ :

$$
\mathcal{H}_{\text {class }}(\vec{s})=\vec{\tau} \cdot \vec{m}(\vec{s})
$$

The components of $\hat{m}(\vec{s})$ are

$E \hat{m}_{3}(\vec{s})=q p_{y} / m, E \hat{m}_{1}(\vec{s})=\frac{q k_{z}}{2 p_{F}^{2}} \frac{\Delta_{0}(r)}{r} y, E \hat{m}_{2}(\vec{s})=-\frac{q k_{z}}{2 p_{F}^{2}} \frac{\Delta_{0}(r)}{r}(r-x)$,

where $E$ is the classical energy: $E^{2}(\vec{s})=\mathcal{H}_{\text {class }}^{2}(\vec{s})$. The $\hat{m}(\vec{s})$ vector field, shown schematically in Fig.3, corresponds to the hedgehog with the $\pi_{2}$ topological charge $1 / 2$ :

$$
N_{\mathrm{zm}}=\frac{1}{8 \pi} \int_{\sigma} d S^{i} e_{i k l}\left(\hat{m} \cdot \frac{\partial \hat{m}}{\partial s_{k}} \times \frac{\partial \hat{m}}{\partial s_{l}}\right)
$$

where the integral is over the hemisphere $\sigma$ about the hedgehhog. This fractional value of the topological invariant $N_{\mathrm{zm}}=1 / 2$, which characterizes the $1 / 2$ vortex, is responsible for the "half-crossing" in correspondence with the index theorem.

\section{Discussion.}

The spectrum of the fermion zero modes in the vicinity of the $1 / 2$ vortices has peculiar topology shown in Fig.1b-c. This follows from the fractional value of the topological invariant, which describes the crossing of the zero energy. This topological invariant coincides with the winding number $N$ of the vortex. For vortices with integer $N$, there are $N$ branches (per spin) which cross zero. For the $N=1 / 2$ vortex, this invariant is fractional and this corresponds to the "half-crossing" in Fig.1b: the spectrum approaches zero but does not cross it, being captured at the zero energy level. Thus far from the half-quantum vortex the spectrum approaches the manifold of the midgap states with zero energy, which is the property of the fermionic bound states in the surface which emanates from the $1 / 2$ vortex. This surface can be the $\pi$-soliton (in the heavy fermionic superconductors) or the Josephson junction with the phase difference $\pi$ (in the high-T superconductors). The existence of the midgap manifold is the robust property of such surfaces [20] 
(see also [21]), in the absence of impurities and if the time inversion symmetry is not violated.

The situation with the zero-energy manifold is similar to the phenomenon of the fermionic condensate discussed in [22, 23] (the extended Van Hove singularity observed in oxide superconductors 24] can be the manifestation of this phenomenon, which is favourable just in the vicinity of the saddle point of spectrum [25]). It is interesting that the existence of the fermionic condensate is also related to the splitting of the $N=1$ vortex into two $N=1 / 2$ vortices but in the momentum $\mathbf{k}$ space [26]. The $N=1$ vortex corresponds to the Fermi-surface in the momentum space. Its splitting results in the Fermiband with zero energy, which appears between the $N=1 / 2$ vortices in the momentum space. In both cases the Fermi-condensate is characterized by a large density of states.

This work was supported through the ROTA co-operation plan of the Finnish Academy and the Russian Academy of Sciences and by the Russian Foundation for Fundamental Sciences.

\section{References}

[1] J.R. Kirtley, C.C. Tsuei, M. Rupp, et al., 1996, Phys. Rev. Lett., 76, 1336 .

[2] V. Geshkenbein, A. Larkin, and A. Barone, 1987,Phys. Rev., B 36, 235.

[3] G.E. Volovik, and V. P. Mineev , Pis'ma Zh. Eksp. Teor. Fiz. 24, 605 (1976) [JETP Lett. 24, 561 (1976)].

[4] M.E. Zhitomirsky, J. Phys. Soc. Jap., 64, 913 (1995).

[5] E. V. Thuneberg, Phys. Rev. Lett. 56, 359 (1986).

[6] M. M. Salomaa and G. E. Volovik, Rev. Mod. Phys., 59, 533 (1987).

[7] G.E. Volovik, Pis'ma Zh. Eksp. Teor. Fiz. 52, 972 (1990) [JETP Lett. 52,358 (1990)].

[8] Y. Kondo, J.S. Korhonen, M. Krusius, V.V. Dmitriev, Yu. M. Mukharskiy, E.B. Sonin and G.E. Volovik, Phys. Rev. Lett. 67, 81 (1991). 
[9] G. Kharadze, and G. Vachnadze, Physica, B 210, 334 (1995).

[10] M.M. Salomaa, G. E. Volovik, J. Low Temp. Phys. 74, 319 (1989).

[11] G.E. Volovik, and L.P. Gor'kov, 1984, Pis'ma Zh. Eksp. Teor. Fiz. 39, 550 [JETP Lett. 39, 674].

[12] M. Sigrist, T.M. Rice, and K. Ueda, 1989, Phys. Rev. Lett. 63, 1727.

[13] M. Sigrist, D.B. Bailey, and R.B. Laughlin, 1995, Phys. Rev. Lett. 74, 3249 .

[14] A.S. Schwarz, Nucl. Phys. B 208, 141 (1982).

[15] M.V. Khazan, Pis'ma Zh. Eksp. Teor. Fiz. 41, 396 (1985) [JETP Lett. 41, 486 (1985)].

[16] J. March-Russel, J. Preskill, and F. Wilczek, 1992, Phys. Rev. D 50, 2567.

[17] A.C. Davis, and A.P. Martin, 1994, Nucl. Phys., B 419, 341.

[18] C. Caroli, P.G. de Gennes, and J. Matricon, Phys. Lett., 9, 307 (1964).

[19] G.E. Volovik, Pis'ma ZhETF, 57, 233 (1993); [JETP Lett., 57, 244 (1993) ]; ZhETF, 104, 3070 (1993); [JETP , 77, 435 (1993) ].

[20] Yu.G. Makhlin, and G.E. Volovik, Pis'ma ZhETF 62 , 719 (1995) [JETP Lett. 62 , 737 (1995) ].

[21] J. Yang, and C.-R. Hu, Phys. Rev., B 50, 16766 (1994).

[22] V.A. Khodel, and V.R. Shaginyan, Pis'ma Zh. Eksp. Teor. Fiz., 51, 488 (1990); [JETP Lett., 51, 553 (1990)].

[23] V.A. Khodel, V.R. Shaginyan, and V.V. Khodel, Phys. Rep., 249, 1 (1994).

[24] T. Yokoya, A. Chainani, T. Takahashi, et al, Phys. Rev. Lett., 76, 3009 (1996). 
[25] G.E. Volovik, Pis'ma ZhETF 59, 798 (1994) [JETP Lett. 59, 830 (1994)].

[26] G.E. Volovik, Pis'ma Zh. Eksp. Teor. Fiz. 53, 208 (1991) [JETP Lett. 53, $222(1991)]$.

Figure 1: Topology of the fermion zero modes in vortices with winding number $N$. (a) In $N=1$ vortex there is one branch of the spectrum, which as a function of the angular momentum $Q$ crosses zero energy. (b) For the vortex with fractional winding number $N=1 / 2$ the spectrum approaches zero but does not cross it, being captured at the zero energy level. (c) The topology of the spectrum in the case of a pair of $N=1 / 2$ vortices, which forms the $N=1$ vortex, is the same as in Fig.1a.

Figure 2: Demonstration of the origin of the spectral asymmetry in the $N=1 / 2$ vortex. If the impact parameter $b$ is positive the quasiparticle trajectory always crosses the surface at which the classical energy takes zero value. For the opposite sign of $b$ the classical energy is always nonzero. 
Figure 3: Index theorem for the $N=1 / 2$ vortex. In the $3 \mathrm{D}$ space of the parameters $\vec{s}=\left(p_{y}, y, x\right)$ there is a line of zeroes of the classical energy, which terminates on the monopole in the field of the unit vector $\hat{m}$, which characterizes the Hamiltonian. The $\pi_{2}$ topological charge of the monopole is fractional and equals $N=1 / 2$. This is the topological origin of the "halfcrossing" of the spectrum in Fig.1b. 